\title{
Informatización de la historia clínica en España
}

\author{
Por Rafael Aleixandre-Benavent, Antonia Ferrer-Sapena y Fernanda Peset
}

\begin{abstract}
Resumen: Se aborda el estado de la informatización de la información sanitaria en España, que cuenta entre sus prioridades con la implantación de una historia de salud electrónica que integre toda la información sobre el estado de salud y de enfermedad de los pacientes, accesible universalmente y que garantice su uso ético, la confidencialidad de la información y la privacidad de las personas. Para ponerla en marcha es imprescindible compatibilizar los sistemas de información de los distintos ámbitos sanitarios actuales, que hasta ahora han funcionado aislados. Aunque las administraciones públicas han puesto en marcha un proyecto de sanidad en línea, todavía persisten las dificultades por la falta de recursos y de infraestructuras y unas barreras entre comunidades autónomas que deben superarse.
\end{abstract}

Palabras clave: Historia de salud, Historia clínica electrónica, Informatización de la información sanitaria, Archivo de imágenes, Sistemas de comunicación, Nomenclatura sistematizada de términos de medicina clínica, Sistema de lenguaje médico unificado.

\section{Title: Electronic medical records in Spain}

Abstract: This paper discusses the current state of computerization of health information in Spain, where the priorities include the establishment of an electronic medical record that integrates all information about patients, makes it universally accessible and ensures the ethical use and confidentiality of the information as well as the privacy of individuals. To implement this change, it is imperative to combine the existing information systems of different health areas that perform in isolation. Although the Spanish gov-

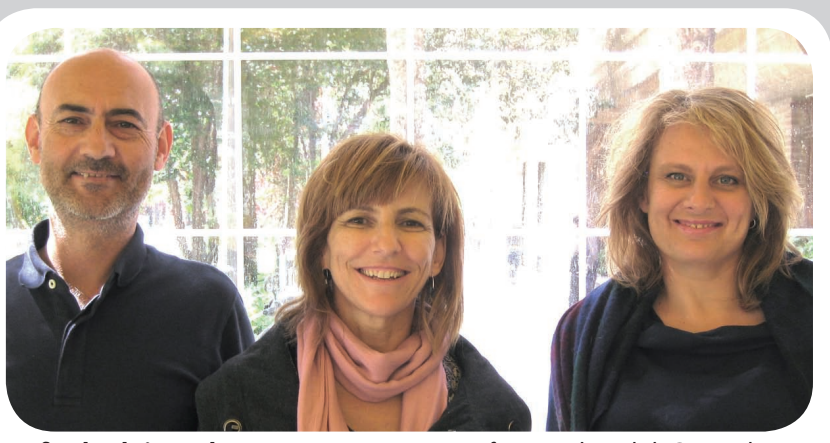

Rafael Aleixandre-Benavent es científico titular del Csic, doctor en medicina y especialista en documentación médica por la Univ. de Valencia. Sus principales líneas de trabajo son la evaluación de la investigación y de las publicaciones científicas, investigación en drogodependencias y estudios sociales sobre inmigración. Ha dirigido numerosas tesis doctorales y proyectos de investigación, y colabora en actividades docentes e investigadoras de varias universidades.

Antonia Ferrer-Sapena es licenciada en geografía e historia en la especialidad de historia contemporánea por la Universidad de Valencia y doctora en técnicas y métodos actuales en información y documentación. Es profesora titular de la Universidad Politécnica de Valencia (UPV) y coordinadora de investigación en Florida Universitaria. De los últimos proyectos dirigidos cabe destacar el portal de economía social EcSocial y la Biblioteca Digital del Cooperativismo Valenciano.

Fernanda Peset es licenciada en geografía e historia y doctora en documentación. Es profesora de la Universidad Politécnica de Valencia (UPV). Sus líneas de investigación se orientan a la comunicación científica, la normalización en la descripción de la información, y el acceso abierto (open access) a la ciencia. Participa en los proyectos IraLIS y E-LIS del Centro Internacional de Investigación en Estrategia y Prospectiva de la Información (Ciepi). Es miembro del think tank ThinkEPI.

http://www.ciepi.org ernment has launched an online health project to achieve this goal, there are still difficulties due to the lack of resources and infrastructure and a few barriers between the Spanish autonomous communities that must be overcome.

Keywords: Health history, Electronic health record, Computerization of health information, Picture archiving and communication systems, Systematized nomenclature of medicine clinical terms, Unified medical language system.

Aleixandre-Benavent, Rafael; Ferrer-Sapena, Antonia; Peset, Fernanda. "Informatización de la historia clínica en España”. El profesional de la información, 2010, mayo-junio, v. 19, n. 3, pp. 231-239.

DOI: 10.3145/epi.2010.may.02

\section{Introducción}

EL AVANCE TECNOLÓGICO está permitiendo la evolución de la información sanitaria tradicional en soporte papel (historia clínica, peticiones, pruebas, resultados, informes, etc.) hacia sistemas electrónicos.
Éstos han de ser capaces de integrar toda la información sobre el estado de salud de los pacientes generada por todos quienes hayan participado en su atención, independientemente de dónde y cuándo haya sucedido. Los sistemas deben ser accesibles universalmente y se debe garantizar su uso ético, la confidencialidad 
de la información y la privacidad de las personas (Altisent-Trota; Delgado-Marroquín, 2004; Red.es, 2009; SEIS, 2002).

La aplicación de las tecnologías de la información y comunicación (TIC) al sector sanitario está más atrasada que en otros campos, como el del comercio o la banca. Esto es debido a que el escenario es demasiado grande para que el sistema nacional de salud pueda acometerla simultáneamente en su totalidad, y a que las TIC han crecido más rápido de lo que los recursos económicos del sistema sanitario han podido asumir (SEIS, 2002).

Además, las infraestructuras básicas necesarias para satisfacer los requerimientos de una actividad tan intensiva en uso de información como la sanitaria son muy numerosas. Podríamos decir que se trata de un sistema de gestión del conocimiento a gran escala, por lo que las dificultades que plantea son múltiples (PonsetiSocías, 2008; Viñeta; Cendra; Salcedo, 2008).

Hasta fechas recientes los recursos invertidos en informatización estaban centrados en las áreas económico-administrativas de los centros, fundamentalmente en la gestión de citas, y se dejaba de lado la gestión de la información clínica. Se echaba en falta una integración entre las prioridades de los principales grupos implicados en la creación de los sistemas de información asistenciales: los gestores sanitarios, cuya principal prioridad es la facturación; los médicos y enfermeros, cuyo principal interés es el acceso a la información clínica; y los informáticos, incapaces muchas veces de comprender la complejidad de la asistencia sanitaria y el adecuado registro, acceso y conservación de la información derivada de esta asistencia (Borrego-Hernando, 2001). Por otra parte, en ningún momento se pensaba en la posibilidad de que el paciente pudiera tener acceso a su propio historial clínico (SEIS, 2002).

\section{"La implantación de la historia de salud electrónica es una de las prioridades de los servicios de salud"}

La situación está cambiando y la implantación de la historia de salud electrónica es una de las prioridades de los servicios de salud en los últimos años (Red.es, 2009). Pero para ponerla en marcha es imprescindible integrar los sistemas de información de los distintos campos sanitarios, que hasta ahora han funcionado aislados. Todas las comunidades autónomas están llevando a cabo la informatización de su sistema sanitario, pero a diferentes velocidades (Cubí-Montfort; Faixe-
das-Brunsoms, 2005; Gómez-Delgado; Serrano-Balazote; Sánchez-Fernández, 2008).

\section{Características de un sistema electrónico de información sanitaria}

El núcleo central del sistema de información es la historia de salud electrónica (HSE) o historia clínica electrónica, cuyo contenido, estructura y funciones están regulados por el artículo 3 de la Ley 41/2002 de 14 de noviembre, básica reguladora de la autonomía del paciente y de derechos y obligaciones en materia de información y documentación clínica (Ley 41/2002). En ella se define la historia clínica como "el conjunto de documentos que contienen los datos, valoraciones e informaciones de cualquier índole sobre la situación y la evolución clínica de un paciente durante el proceso asistencial. Está constituida por el conjunto de documentos, tanto escritos como gráficos, que hacen referencia a los episodios de salud y enfermedad de una persona, y a la actividad sanitaria que se genera con motivo de esos episodios" (Alventosa-del-Río, 2003; García-Ortega; Cózar-Murillo; Almenara-Barrios, 2004; Jiménez-Pérez, 2001).

El concepto de HSE es mucho más amplio que el de la historia clínica tradicional, pues es el registro cronológico de todos los acontecimientos relativos a la salud de una persona, tanto preventivos como asistenciales, desde el nacimiento e incluso antes, hasta su fallecimiento. Incluye la historia de atención primaria y todos los episodios concretos de atención especializada, aspectos no incluidos en la historia clínica tradicional (SEIS, 2002).

Para hacerse una idea de la complejidad que acarrea un proceso sistemático de automatización de la información sanitaria, vale la pena revisar brevemente sus principales características:

\section{"La historia de salud electrónica es el registro cronológico de todos los acontecimientos de la salud de una persona"}

En primer lugar, debe integrar información procedente de los diversos niveles:

- Asistenciales: atención primaria, especializada y hospitalaria, tanto pertenecientes al sistema público como al privado o concertado, incluyendo la hospitalización a domicilio y los servicios de urgencias.

- Departamentales: laboratorios, farmacia, diagnóstico por imagen, gestión económico-financiera. 
- Sistemas complementarios al clínico: salud laboral, programas de promoción de la salud y prevención de la enfermedad, prestaciones sanitarias complementarias.

- Información procedente del sistema de receta electrónica y de los sistemas de ayuda a la toma de decisiones clínicas.

En segundo lugar, se trata de una información que es manejada por múltiples usuarios:

- Miembros de los equipos de salud (médicos, enfermeros, fisioterapeutas, etc.), pues su función principal es la asistencia sanitaria y el mantenimiento y la promoción de la salud.

- Gestores, que precisan datos que permitan la planificación de los recursos y la valoración de resultados.

- Administradores, que necesitan información sobre los tratamientos y exploraciones para su facturación y sobre la gestión de pacientes, tanto hospitalizados como ambulatorios.

- Investigadores, profesores y estudiantes de las ciencias de la salud.

- Funcionarios de justicia, cuando se presentan asuntos jurídico-legales relacionados con la asistencia sanitaria. La normativa legal autoriza la cesión o comunicación de los datos de la historia clínica, aun sin consentimiento del interesado, a jueces y tribunales, el Defensor del Pueblo, el Ministerio Fiscal y el Tribunal de Cuentas.

- Auditores, para el control de la calidad asistencial.

- Servicios sociales, para hacer una valoración de las capacidades del individuo y su contexto.

- Servicios de salud pública, para poder realizar estudios epidemiológicos y emprender actuaciones preventivas (por ejemplo las vacunaciones).

- Ciudadanos, quienes han dejado de ser un elemento pasivo del proceso sanitario y tienen derecho al acceso a los datos de la historia clínica, con excepción de los confidenciales que afecten a terceras personas.

Es necesario definir previamente qué información necesita cada grupo de usuarios y en qué formato. No todos los grupos pueden disponer de los mismos datos por motivos de confidencialidad. En todos los casos, cualquier acceso deberá ser autentificado y quedará identificado y registrado en el correspondiente documento de seguridad. Además, deberá existir un registro que conserve la información de detalle relativa a cada acceso (Altisent-Trota; Delgado-Marroquín, 2004).

\begin{tabular}{|c|c|}
\hline Historia clínica en papel & Historia de salud electrónica \\
\hline $\begin{array}{l}\text { Desorden de los documentos, desorganización y fragmen- } \\
\text { tación de la información }\end{array}$ & $\begin{array}{l}\text { Ordenación de la información de la forma que convenga a } \\
\text { la persona que accede a la misma. Organización preesta- } \\
\text { blecida y flexibilidad en la visualización }\end{array}$ \\
\hline $\begin{array}{l}\text { Dificultades en la recuperación de la información contenida } \\
\text { en estos documentos de una forma racional y lógica }\end{array}$ & $\begin{array}{l}\text { Fácil recuperación de la información y posibilidad de com- } \\
\text { binar criterios de búsqueda y operadores }\end{array}$ \\
\hline Falta de uniformidad de los documentos & Mayor uniformidad en los documentos \\
\hline Ilegibilidad de los documentos manuscritos & Legibilidad absoluta \\
\hline Dudosa confidencialidad por falta de protección & $\begin{array}{l}\text { Control y registro de todos accesos sin excepción. } \\
\text { Habilitación de permisos en las debidas circunstancias }\end{array}$ \\
\hline Alterabilidad & $\begin{array}{l}\text { Inalterabilidad. Cualquier modificación queda registrada } \\
\text { con la fecha y autor de la misma. Seguridad de los datos }\end{array}$ \\
\hline $\begin{array}{l}\text { Disponibilidad cuestionable. No es posible acceder simultá- } \\
\text { neamente a la misma historia clínica por dos o más perso- } \\
\text { nas desde diferentes lugares }\end{array}$ & $\begin{array}{l}\text { Disponibilidad total y accesibilidad inmediata y concurrente } \\
\text { desde cualquier lugar y tiempo }\end{array}$ \\
\hline Errores de archivado que impiden su localización & Sin errores de archivado \\
\hline $\begin{array}{l}\text { Deterioro del soporte por los medios físicos (agua, hume- } \\
\text { dad, calor, agentes corrosivos, fuego...) }\end{array}$ & $\begin{array}{l}\text { Garantía del soporte: puede preservarse el soporte docu- } \\
\text { mental del riesgo de accidentes mediante copias de seguri- } \\
\text { dad y mecanismos en espejo (duplicidad de servidores) }\end{array}$ \\
\hline $\begin{array}{l}\text { Difícil separación de los datos de identificación del paciente } \\
\text { de los de carácter clínico-asistencial en los supuestos de } \\
\text { acceso diferentes a los asistenciales y judiciales }\end{array}$ & Fácil separación datos \\
\hline $\begin{array}{l}\text { Necesita mucho espacio para su almacenamiento así como } \\
\text { personal para su gestión }\end{array}$ & $\begin{array}{l}\text { Almacenamiento de la información en soportes electrónicos } \\
\text { que minimizan el espacio y el personal necesario para su } \\
\text { gestión }\end{array}$ \\
\hline Imposible integración con otros sistemas & Posibilidad de integración con otros sistemas electrónicos \\
\hline
\end{tabular}

Tabla 1: Ventajas de la historia de salud electrónica frente a la historia clínica en papel 
En tercer lugar, el sistema debe caracterizarse por su gran capacidad de almacenamiento, ya que debe albergar miles de documentos y pruebas en formatos de alta resolución. Debe asegurar un alto nivel de accesibilidad desde cualquier lugar del centro sanitario o de otros centros y en cualquier momento; y también garantizar la portabilidad para que un médico que pasa visita en una sala de un hospital pueda consultar o introducir los datos que necesite.

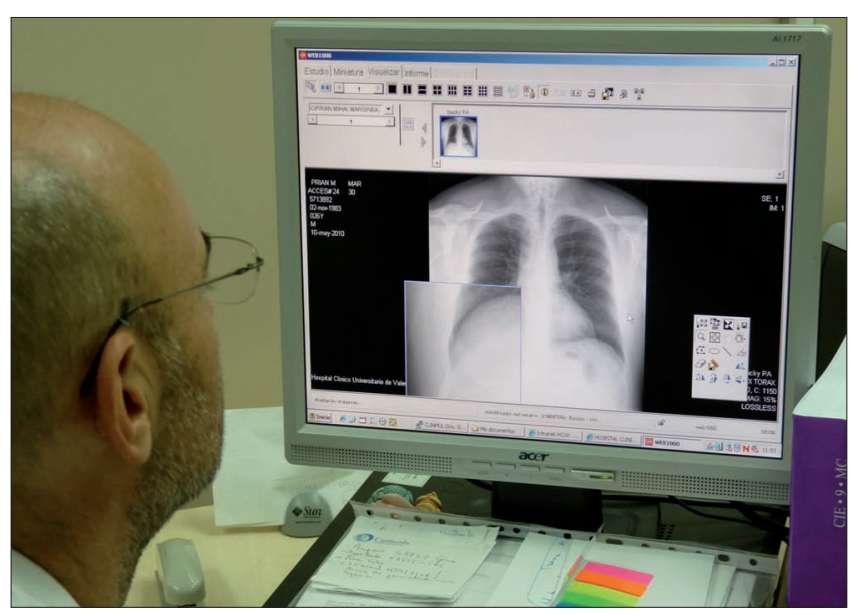

\section{Ventajas de un sistema automatizado de información sanitaria}

Los principales problemas que plantea la información sanitaria en soporte papel son bien conocidos: falta de uniformidad entre los modelos utilizados en los diversos centros, desorden propio de un expediente que es manejado por múltiples usuarios, ilegibilidad de la información escrita a mano, accesibilidad, errores en el archivado, necesidad de espacio para su almacenamiento, posibilidad de que su contenido pueda alterarse, perderse o deteriorarse, y dudosas garantías de confidencialidad. Como puede apreciarse en la tabla 1, la historia de salud electrónica resuelve todos estos problemas de manera eficiente y aporta ventajas añadidas (SEIS, 2002).

Una de ellas es la automatización de numerosas tareas que producen retrasos. Cabe citar como ejemplo la gestión automatizada de peticiones clínicas. Cuando un clínico hace una petición a un servicio, por ejemplo al de Radiología, la petición llega automáticamente en tiempo real y, cuando ésta es programada o resuelta, se comunica directamente al emisor de la petición. Así desaparecen los traslados de papel por parte de celadores. Otro ejemplo más sofisticado es la automatización del sistema de dispensación de medicamentos, que permite conocer el estado actual de medicación de los pacientes.

Disponer de toda la información del paciente en cualquier lugar produce cambios en los métodos tra- dicionales de trabajo. Los formularios impresos están siendo sustituidos por dispositivos portátiles donde los médicos registran la evolución de sus pacientes en la propia habitación del centro. El uso de asistentes digitales (pda) y pizarras digitales (tablet pc) evita el papel y las posteriores transcripciones, lo que mejora la calidad de la atención (Alegre; Cabrer, 2005). Otros recursos como las pantallas gráficas en urgencias y en las salas informan del estado de ocupación de las camas simulando el corcho tradicional. Es posible también tener localizados a los pacientes y monitorizar constantemente el estado de sus peticiones, así como conocer al momento si los resultados están disponibles y su evolución durante la estancia en urgencias o en el hospital (Ponseti-Socías, 2008; Ramos-López; Cuchí-Alfaro; Sánchez-Molano, 2009).

Otra ventaja de la automatización de la información sanitaria se refiere a la integración de datos capturados directamente desde dispositivos. Tanto los autoanalizadores de laboratorio como los electrocardiógrafos inalámbricos transmiten los resultados por la red por lo que pueden ser consultados en todo el sistema. Se utilizan otros sistemas de captura directa como la lectura de códigos de barras y los procedentes de los PACS (picture archiving and communication systems). Estos últimos son repositorios digitales dedicados al almacenamiento, recuperación, distribución y presentación de imágenes médicas electrónicas en cualquier formato, no sólo radiológicas sino de todas las imágenes que integran las historias clínicas (endoscópicas, ecográficas, oftalmológicas, dermatológicas, de anatomía patológica, etc.), y pueden o no ir acompañadas de un informe (Ulecia-Martínez et al., 2007).

Los sistemas de información automatizados pueden incluir también aplicaciones de soporte a las decisiones

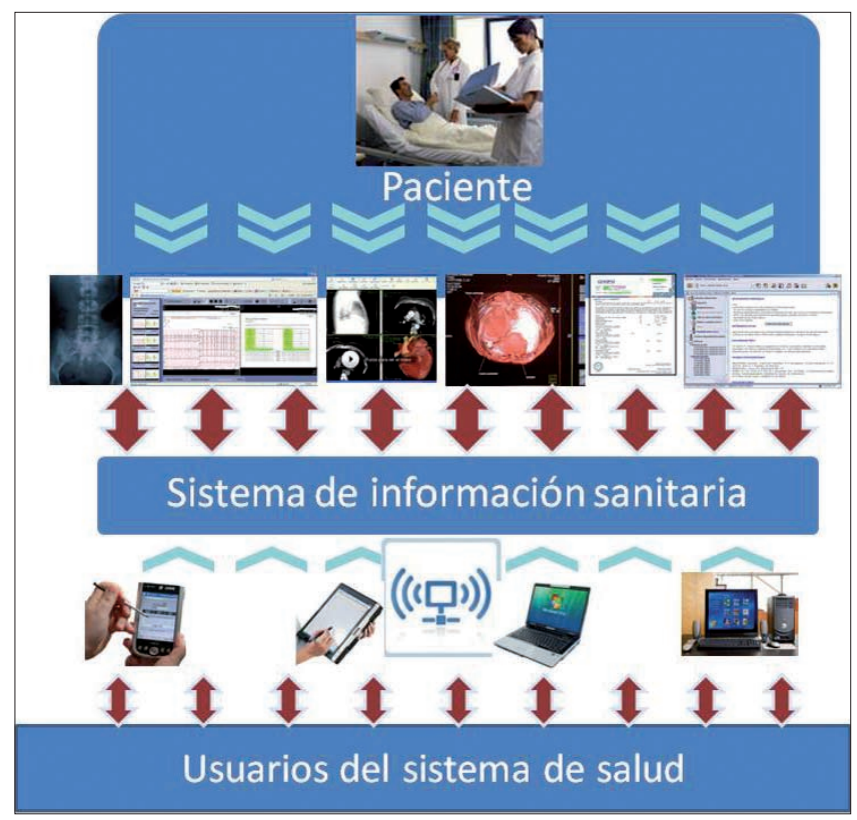


médicas, como los vademecums, que proporcionan información clínica y farmacológica, y otras herramientas de utilidad clínica como guías terapéuticas, calculadora de dosis pediátricas, tablas de administración de fármacos de extrema urgencia, etc. (Llopis-Salvia; SánchezAlcaraz; Quintana-Vergara, 2003). Se acompañan de otras utilidades como los módulos de seguridad para evitar errores en la prescripción mediante la detección automática de duplicidades, interacciones medicamentosas e incompatibilidades, la emisión de alertas de errores de dosis o dosificación inadecuada para la situación clínica del paciente, y la ayuda en línea para la consulta de posologías y de otras características farmacológicas (Bobb et al., 2002). Se ha demostrado que la prescripción informatizada puede prevenir el $64 \%$ de los errores de prescripción, de los cuales el $43 \%$ son potencialmente dañinos, y previene accidentes por alergia o interacciones medicamentosas gracias a estas alertas (Giménez-López; Navarro-Pemán; LanuzaGiménez, 2006).

No menos importantes son los directorios de médicos y de centros sanitarios, la conexión con laboratorios de análisis clínicos para efectuar peticiones de pruebas y recepción y consulta de resultados o la posibilidad de realizar interconsultas directas con profesionales de otros centros sanitarios pudiendo intercambiar cualquier tipo de datos, imagen o información con ellos de manera transparente y quedando registrada en el sistema. El enlace a repositorios de información bibliográfica científica también resulta de interés para el ejercicio profesional (Abad-García et al., 2008), así como el acceso a las infografías para la comunicación en el sector de la salud (González-Pacanowski; Medina, 2009).

\section{"La historia de salud electrónica (HSE) es una pieza clave en la investigación y enseñanza de las ciencias de la salud"}

Por último, el potencial informativo de la HSE es tal que se convierte en una pieza clave en la investigación y enseñanza de las ciencias de la salud. En relación con la investigación hace posible, por ejemplo, establecer un conjunto de bases de datos estandarizadas de enfermedades y procesos con datos integrados procedentes de múltiples centros, lo que permite su comparación, la observación de desviaciones y la valoración objetiva de los resultados obtenidos. En cuanto a la enseñanza, profesores y estudiantes disponen de un instrumento de gran valor informativo y educativo con el que recoger experiencia y aprendizaje, a la vez que les otorga una formación continua individualizada. Algunas de las nuevas herramientas docentes que proporciona la HSE son: bases de datos de imágenes sobre enfermedades, lesiones, procedimientos diagnósticos e intervenciones quirúrgicas; herramientas de ayuda a la decisión; simuladores de pacientes y realidad virtual alimentados con información procedente de la HSE.

Hasta ahora hemos reflejado la situación que podría considerarse ideal para un sistema de información sanitario, teniendo en cuenta las posibilidades de las nuevas tecnologías, si los recursos asignados fueran suficientes. Pasaremos ahora a describir una realidad, la española.

\section{Situación de la historia clínica electrónica en España}

La incorporación de las TIC en el sector sanitario se ha llevado a cabo paulatinamente pero sin planificación de conjunto. Como resultado han aparecido múltiples subsistemas aislados, procedentes de diferentes proveedores, tecnologías e incluso de diferentes eras informáticas, con información duplicada, inconsistente y en muchos casos no accesible desde todo el hospital y mucho menos desde todo el distrito sanitario, la comunidad autónoma o el país (Campillo-Artero, 2008).

No hay que olvidar que alrededor de cuatro millones de españoles requieren atención médica fuera de su comunidad autónoma cada año. Aunque la mayoría de ellos no precisa disponer de información sobre sus antecedentes clínicos para recibir una asistencia correcta, en otros casos estos datos resultan clave (GonzálezCocina; Pérez-Torres, 2007).

\section{"La fragmentación española en comunidades autónomas y la falta de liderazgo del $\mathbf{M}^{\circ}$ de Sanidad, han resultado negativas para la implementación de la historia clínica electrónica"}

Se estima que sólo 50.000 médicos españoles (aproximadamente el 30\% de los colegiados) trabajan con HSE y, además, utilizan un gran número de diferentes modelos de software. Cada una de las 17 comunidades autónomas españolas cuenta con un modelo diferente de sistema de información sanitaria y de tarjeta sanitaria. En la mayoría de ellas hay incluso diferentes modelos de HSE para hospitales y para atención primaria sin conectividad entre ellos. La situación es similar en los sistemas de información de la sanidad privada, 
donde tampoco existe ninguna conectividad entre ellos ni con la pública.

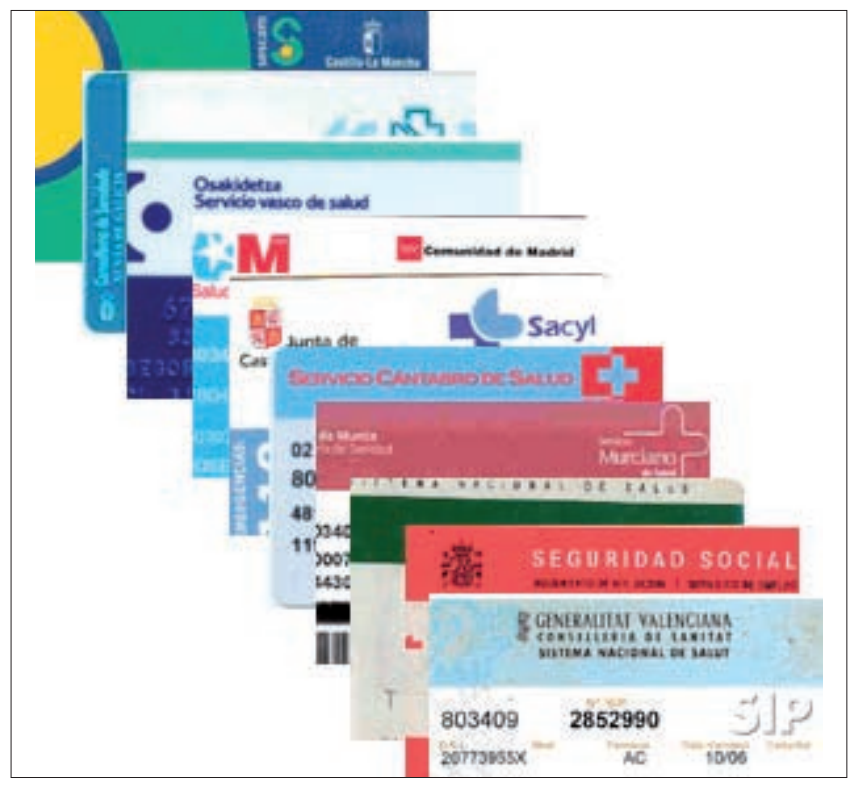

Tarjetas sanitarias distintas para cada comunidad autónoma española

Esta falta de integración e interoperabilidad producida por la forma en que se ha implantado la tecnología da cuenta de la ineficacia del sistema en su globalidad. La fragmentación española en comunidades autónomas y la falta de liderazgo del $\mathrm{M}^{\mathrm{o}}$ de Sanidad, han resultado nefastos para la implementación de la historia clínica electrónica. Para tratar de resolver este problema en 2006 se inició el proyecto Sanidad en Línea (Red.es, 2009), dentro de la iniciativa gubernamental del Plan Avanza, en el que colaboran el Ministerio de Industria, Turismo y Comercio, el Ministerio de Sanidad y Política Social y las comunidades autónomas.

http://www.planavanza.es/Paginas/Inicio.aspx

Según el informe preliminar, el 96\% de los centros de salud disponen de una solución informática, el 65\% ofrece un servicio de citas por internet y el $97 \%$ de los hospitales dispone de un sistema de información para la gestión de las admisiones, camas, agenda y citas de consultas externas. El 85\% tiene un sistema de gestión de radiología, anatomía patológica y farmacia. Los citados pacs están disponibles en el $62 \%$ de los hospitales españoles y el $90 \%$ cuenta con un sistema de información de laboratorio (Red.es, 2009).

Las comunidades autónomas de Andalucía, Baleares, Castilla La Mancha, Catalunya, Comunidad Valenciana, Extremadura, Navarra y País Vasco han finalizado su implantación de la HSE y su integración con los centros de salud. Este adelanto hace posible que los profesionales de atención primaria puedan acceder directamente desde sus consultas a la información sobre el paciente que hayan registrado otros médicos. La receta electrónica está implantada en Andalucía y Baleares y las otras comunidades autónomas están trabajando en ello. También la cita telemática por internet se encuentra en fase avanzada en la mayor parte de autonomías.

El proyecto tiene como finalidad llevar a cabo al menos cinco grandes líneas de actuación para apoyar el trabajo de los profesionales sanitarios en beneficio del ciudadano:

- Un sistema fiable de identificación unívoca de los usuarios (tarjeta sanitaria individual).

- La informatización de los registros clínicos de cada usuario o paciente (historia clínica digital).

- Un sistema que facilite la prestación farmacéutica a los pacientes y usuarios (prescripción, visado y dispensación de medicamentos).

- Nuevos servicios de relación entre los ciudadanos y los profesionales: cita por internet, telemedicina y teleformación.

- Garantizar la accesibilidad desde cualquier punto del sistema, la interoperabilidad y la explotación adecuada de la información.

Además, el programa plantea los siguientes objetivos básicos de informatización:

- Incorporar las tarjetas sanitarias de las comunidades autónomas a la base de datos común del Sistema Nacional de Salud.

- Apoyar a las comunidades autónomas para implantar la historia clínica digital en su territorio, con elementos de interoperabilidad con el Sistema Nacional de Salud.

- Extender el sistema de receta electrónica para la prescripción y dispensación de medicamentos a todo el territorio nacional.

- Fortalecer el Nodo Central de Servicios del Sistema Nacional de Salud, apoyando y garantizando la interoperabilidad de los diferentes sistemas.

\section{Retos pendientes}

En encuestas realizadas sobre las ventajas e inconvenientes de la HSE (Alonso et al., 2004; Chaudhry et al., 2006; Walsh, 2004; Olvera-Lobo; Benítez-deVandrell, 2008), los médicos manifestaron una opinión favorable, con un alto grado de utilización de las herramientas, si bien con el inconveniente de las dificultades para el correcto manejo del programa. Añadían que para que la informatización sea aceptada debe facilitar el trabajo sin influir negativamente en la relación médico-paciente, la calidad de la atención y la confidencia- 
lidad. El análisis de las opiniones vertidas en las encuestas realizadas a los usuarios lleva a plantear la necesidad de que las organizaciones dirijan sus esfuerzos a combatir las percepciones negativas originadas al implantar la informatización de la información sanitaria, debidas sobre todo a su falta de conocimientos informáticos y de formación específica en el manejo de los programas. Se ha demostrado que a menor conocimiento informático mayor rechazo a la utilización de una HSE frente a una historia clínica en papel (Walsh, 2004).

Una de las principales novedades de los sistemas electrónicos es que por primera vez el usuario podrá acceder a da-

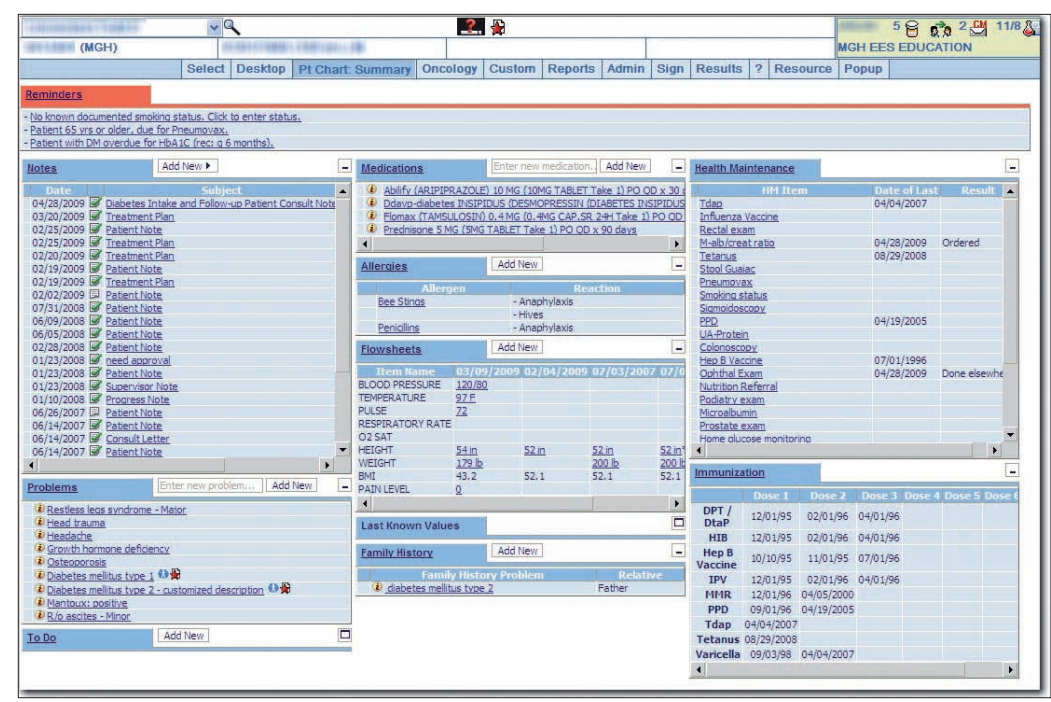

Ejemplo de historia clínica electrónica (electronic medical record, EMR) del Martha's Vineyard Hospital, Massachusetts tos básicos sobre su salud a través de internet y será él quien autorice y controle quién más puede consultarlos. La información accesible será limitada; es decir, se tratará de una historia clínica resumida que incluirá diversos informes, como los de alta de hospitalización, atención primaria, urgencias, consulta de enfermería, resultados de pruebas de imagen, laboratorio y otras pruebas diagnósticas, lo que en algunos sistemas se conoce como "carpeta personal de salud".

\section{"El usuario podrá acceder a datos básicos sobre su salud a través de internet y será él quien autorice y controle quién más puede consultarlos"}

Un problema añadido que debe resolverse es la falta de interoperabilidad semántica producida porque no todos los documentos se encuentren escritos en el mismo idioma. Si se quiere que la historia clínica del futuro sea accesible en cualquier parte del mundo, va a ser necesaria una sintaxis común, una terminología clínica inteligible a todo profesional sanitario sea cual sea la nación en la que trabaje. Hay dos proyectos de lenguajes documentales en marcha para la HSE: el UMSL (Unified medical language system) y el Snomed-CT (Systematized nomenclature of medicine clinical terms) (Fenton, 2005; Rosenbloom et al., 2006). El UMSL es la compilación e integración de los lenguajes documentales más importantes usados en medicina y pretende facilitar el acceso a diferentes aplicaciones (HCE, bases de datos bibliográficas, sistema de ayuda a la decisión clínica). La International Health Terminology Standards Development Organization trabaja desde hace años en la elaboración de un lenguaje médico codificado y en un sistema capaz de reconocer y traducir los idiomas locales. Desde 2007 explota y administra los derechos de Snomed-CT, considerada la terminología médica multilingüe más amplia, que incluye más de 31.000 conceptos y 800.000 descripciones (Cornet; De-Keizer, 2008). España se ha incorporado en 2009 (Red.es, 2009).

http://www.ihtsdo.org/

http://www.ihtsdo.org/snomed-ct/

\section{Conclusiones}

Los sistemas digitales de información sanitaria se caracterizan por su diversidad y por la elaboración de numerosas aplicaciones informáticas individuales no integradas con otros sistemas, lo que les confiere una escasa compatibilidad y unas condiciones de seguridad que generan dudas sobre las garantías de confidencialidad de los datos personales almacenados (Grupo de trabajo de bioética de la Semfyc, 2004; Júdez et al., 2002; De-Miguel-Sánchez, 2006).

La implantación de la HSE, las técnicas digitales empleadas en la grabación de numerosas pruebas diagnósticas, el uso de dispositivos móviles inalámbricos desde los que los profesionales médicos tienen acceso a los datos del paciente desde cualquier sitio del centro y la posibilidad de acceder a información online durante la consulta como soporte a sus decisiones, están mejorando la eficiencia de los profesionales y la calidad del servicio prestado a los pacientes.

Por otra parte, se ha demostrado una reducción de costes por la disminución en la petición de pruebas y exámenes, pues las herramientas de ayuda se utilizan más, por la disponibilidad de las pruebas anteriores y por el sistema computerizado de alertas (Chaudhry et al., 2006). 


\section{"Existe un consenso general sobre los beneficios de la HSE pero todavía persisten las dificultades por la falta de recursos"}

En definitiva, si bien existe un consenso general sobre los beneficios de la HSE, que pueden resumirse en la posibilidad de que exista una historia única, su interoperabilidad con otros sistemas, su disponibilidad, su mayor seguridad y confidencialidad, su papel en la mejora de la calidad y de la eficiencia del proceso asistencial, todavía persisten las dificultades, normalmente debidas a la falta de recursos, y unos retos pendientes que pueden sintetizarse en (Red.es, 2009):

- Acelerar los plazos para que los ciudadanos se beneficien de las ventajas de la sanidad electrónica.

- Garantizar la interoperabilidad tecnológica en las tres grandes áreas de actuación: tarjeta sanitaria individual, HCE y receta electrónica.

- Disminuir las barreras entre las comunidades autónomas.

- Reforzar las infraestructuras.

\section{Bibliografía}

Abad-García, María-Francisca; González-Teruel, Aurora; MartínezCatalán, Celeste; Giménez-Martínez, Francesc. "Viabilidad de repositorios de biomedicina y ciencias de la salud en la Comunidad Valenciana". El profesional de la información, 2008, marzo-abril, v. 17, n. 2, pp. 165-173.

Alegre, Luis; Cabrer, Miguel. "El Hospital Son Llàtzer como plataforma para proyectos eSalud". RevistaeSalud.com, 2005, v. 1, n. 2.

http://www.revistaesalud.com/index.php/revistaesalud/article/view/17/17

Alonso-Sánchez, Ángel; Iraburu, Marga; Saldaña, M. Lourdes; De-Pedro-Montalbán, M. Teresa. "Implantación de una historia clínica informatizada: encuesta sobre el grado de utilización y satisfacción en un hospital terciario". Anales del Sistema Sanitario de Navarra, 2004, v. 27, n. 2, pp. 233-239.

http://recyt.fecyt.es/index.php/ASSN/article/view/4910/4167

Alventosa-del-Río, Josefina. "La Ley 41/2002 de 14 de noviembre, básica reguladora de la autonomía del paciente y de los derechos y obligaciones en materia de información y documentación clínica (y II)". Revista española de drogodependencias, 2003, v. 28, n.3, pp. 384-401.

Altisent-Trota, Rogelio; Delgado-Marroquín, María-Teresa. "La doble faz de la técnica: informatización y confidencialidad en la historia clínica". Atención primaria, 2004, v. 34, n. 3, pp. 115-116.

Bobb, Anne; Gleason, Kristine; Husch, Maria; Feinglass, Joe; Yarnold, Paul; Noskin, Gary. "The epidemiology of prescribing errors. The potential impact of computerized prescriber order entry". Archives of internal medicine, 2004, v. 164, pp. 785-792.

Borrego-Hernando, Leopoldo. "Aplicaciones informáticas en la consulta de dermatología. Programas de gestión de consultas. Historia clínica informatizada. Bases de datos". Piel, 2001, v. 16, n. 3, pp. 125-131.

Campillo-Artero, Carlos. "Fracturas en la informatización de los servicios de salud". Gaceta sanitaria, 2008, v. 22, n. 4, pp. 371-377.

http://scielo.isciii.es/scielo.php?pid=S0213-91112008000400012\&script $=$ sci_arttext
Chaudhry, Basit; Wang, Jerome; Wu, Shinyi; Maglione, Margaret; Mojica, Walter; Roth, Elizabeth; Morton, Sally C.; Shekelle, Paul. "Impact of health information technology on quality, efficiency, and costs of medical care". Annals of internal medicine, 2006, v. 144, n. 10, pp. 742-752. http://www.annals.org/content/144/10/742.full

Cornet, Ronald; De-Keizer, Nicolette. "Forty years of Snomed: a literature review". BMC medical informatics and decision making, 2008, v. 8, Suppl. 1: S2.

http://www.biomedcentral.com/1472-6947/8/S1/S2

Cubí-Montfort, Rafel; Faixedas-Brunsoms, Delfí. "Avances en la informatización de los sistemas de salud". Atención primaria: publicación oficial de la Sociedad Española de Familia y Comunitaria, 2005, v. 36, n. 8, pp. 448-452.

De-Miguel-Sánchez, Noelia. "Investigación y protección de datos de carácter personal: una aproximación a la ley 14/2007, de 3 de julio, de investigación biomédica". Revista española de protección de datos, 2006, v. 1, pp. 143-201.

Fenton, Susan H. "An introduction to the Unified Medical Language System". Journal of the American Health Information Management Association, 2005, v. 76, n. 5, pp. 60-64.

García-Ortega, Cesáreo; Cózar-Murillo, Victoria; Almenara-Barrios, José "La autonomía del paciente y los derechos en materia de información y documentación clínica en el contexto de la Ley 41/2002". Revista española de salud pública, 2004, v. 78, n. 4, pp. 469-479.

http://redalyc.uaemex.mx/redalyc/pdf/170/17078405.pdf

Giménez-López, Virginia; Navarro-Pemán, Cristina; Lanuza-Giménez, Javier. "Análisis de las notificaciones de reacciones adversas a medicamentos tras la informatización de las consultas de atención primaria en Aragón". Archivos de la Facultad de Medicina de Zaragoza, 2006, v. 46, n. 1, pp. 7-13.

Giménez-Pérez, Dolors. "La historia clínica: aspectos éticos y legales". Revista de calidad asistencial, 2001, v. 16, pp. 66-68.

Gómez-Delgado, Ángel; Serrano-Balazote, Pablo; Sánchez-Fernández, Ana-María. "Historia clínica electrónica: de la integración a la interoperabilidad". Todo hospital, 2008, n. 248, pp. 421-427.

González-Cocina, Emilio; Pérez-Torres, Francisco. "La historia clínica electrónica. Revisión y análisis de la actualidad. Diraya: la historia de salud electrónica de Andalucía”. Revista española de cardiología, 2007, v. 7, supl. C, pp. 37-46.

González-Pacanowski, Toni; Medina, Pablo. "Comunicación online en el sector salud. Valor de la infografía”. El profesional de la información, 2009, julio-agosto, v. 18, n. 4, pp. 413-420.

Grupo de trabajo de bioética de la Sociedad Española de Medicina Familiar y Comunitaria. "Informatización y confidencialidad de la historia clínica”. Atención primaria, 2004, v. 34, n. 3, pp. 140-142.

Júdez, Javier; Nicolás, Pilar; Delgado, María-Teresa; Hernando, Pablo; Zarco, José; Granollers, Silvia. "La confidencialidad en la práctica clínica: historia clínica y gestión de la información”. Medicina clínica, 2002, v. 118, n. 1 , pp. 18-37.

Ley 41/2002, de 14 de noviembre, básica reguladora de la autonomía del paciente y de derechos y obligaciones en materia de información y documentación clínica. BOE n. 274, pp. 40126-40132.

Llopis-Salvia, Pilar; Sánchez-Alcaraz, Agustín; Quintana-Vergara, Belén. "Informatización integral de la asistencia sanitaria en el paciente hospitalizado. Repercusión sobre las actividades de atención farmacéutica". Farmacia hospitalaria, 2003, v. 27, n. 4, pp. 231-239.

http://www.elsevier.es/revistas/ctl_servlet?_f $=7016$ \&articuloid $=13118810$ $\&$ revistaid $=121$

Olvera-Lobo, María-Dolores; Benítez-de-Vandrell, Belarmina. “Aproximación a las actitudes y percepciones de los usuarios ante las tecnologías de la información". El profesional de la información, 2008, v. 17, n. 2, pp. 199-204.

Ponseti-Socías, María. "Experiencia de las TIC en el Hospital Son Llátzer". Todo hospital, 2008, n. 248, pp. 387-397.

Ramos-López, Juan-Manuel; Cuchí-Alfaro, Miguel; Sánchez-Molano, María-Ángeles. "Archivo de historias clínicas digitalizado, una solución 
previa a la historia clínica electrónica”. Papeles médicos, 2009, v 18, n. 2 , pp. 4-8.

http://www.sedom.es/3_papeles/18_2/18_2_articulo1.pdf

Red.es (ed.). Las TIC en el Sistema Nacional de Salud. El programa Sanidad en Línea. Madrid, 2009.

http://www.ontsi.red.es/sanidad/articles/id/3032/las-tic-el-sistemanacional-salud.html

Rosenbloom, S. Trent; Miller, Randolph A.; Johnson, Kevin B.; Elkin, Peter L.; Brown, Steven H. "Interface terminologies: facilitating direct entry of clinical data into electronic health record systems". Journal of the American Medical Informatics Association, 2006, v. 13, n. 3, pp. 277-288.

SEIS (Sociedad Española de Informática de la Salud). Informes SEIS. De la historia clínica a la historia de salud electrónica. Pamplona, 2003, n. 5. http://www.conganat.org/seis/informes/2003/

Ulecia-Martínez, Miguel-Ángel; González-Cocina, Emilio; MontijanoCabrera, Ángela M.; Caballero-Güeto, Julia; Gómez-Doblas, JuanJosé; De-Mora-Martín, Manuel. "Instrumentos de mejora en la calidad asistencial para las enfermedades cardiovasculares en el siglo XXI: análisis de la historia clínica electrónica y de consultas de alta resolución". Revista andaluza de cardiología, 2007, v. 42, n. 4, pp. 255-263.

http://www.sacardiologia.org/pdf/revistas/5657ca4318989c93f26092 af779d390a.pdf

Viñeta, Maite; Cendra, Carlos; Salcedo, J. Luis. "Papel de las TIC en el hospital en transformación: estrategias para su implantación". Todo hospital, 2008, n. 248, pp. 380-386
Walsh, Stephen H. "The clinician's perspective on electronic health records and how they can affect patient care". British medical journal, 2004, v. 328 , pp. $1184-1187$

\section{Agradecimientos}

Los autores agradecen a María T. Moreno-Núñez, Antonia Agulló-Martínez, Begoña Cantos-Aldaz e Isabel Iniesta sus aportaciones y ayuda.

Rafael Aleixandre-Benavent, Instituto de Historia de la Medicina y de la Ciencia López Piñero. Unidad de Información e Investigación Social y Sanitaria (Uisys), Universitat de València-CSIC, Valencia.

Rafael.Aleixandre@uv.es

Antonia Ferrer-Sapena, Fernanda Peset. Departamento de Comunicación Audiovisual, Documentación e Historia del Arte. Universidad Politécnica de Valencia anfersa@upv.es

mpesetm@upv.es

\section{ProQuest}

\section{Ciencias de la Salud}

Colecciones de revistas y libros electrónicos para bibliotecas académicas, hospitalarias, públicas y especializadas.

- Todas las disciplinas clínicas y biomédicas

- Ciencias de la Salud y disciplinas afines

- Medicina Basada en Evidencia

- Enfermería Basada en Evidencia

- Medicina Familiar

- Administración de la Salud

- Enfermería

- Farmacia

Para cualquier consulta, póngase en contacto con editor@spain.proquest.com con la referencia AD 69610 ó visite www.proquest.co.uk/go/healthsciences 